\title{
Measurements of vector boson fusion with the ATLAS detector
}

\section{Chris Hays*}

Oxford University

On behalf of the ATLAS collaboration

E-mail: chris.hays@physics.ox.ac.uk

\begin{abstract}
Measurements are presented of single $W$ and $Z$ boson production in a vector-boson-fusion topology using ATLAS data collected at the LHC. The measurements include the fiducial cross sections in $\sqrt{s}=7$ and $8 \mathrm{TeV} p p$ collisions of $W$ and $Z$ production with jets produced via the electroweak interaction, of which vector-boson fusion is a component, and with jets produced via the strong process. Differential cross section measurements are also shown.
\end{abstract}

XXV International Workshop on Deep-Inelastic Scattering and Related Subjects 3-7 April 2017

University of Birmingham, $U K$

${ }^{*}$ Speaker. 


\section{Introduction}

Vector-boson fusion processes are an important tool to probe for new particles, particularly those that do not have colour charge and can only be produced via electroweak processes. The large cross sections and clean signatures of $W \rightarrow \ell v$ and $Z \rightarrow \ell \ell$ production at the LHC allow the most precise probes of electroweak processes in a vector-boson fusion (VBF) topology. Measurements of these cross sections identify the leading uncertainties associated with this topology, relevant in particular for Higgs boson production, and are directly sensitive to anomalous triplegauge couplings.

Cross sections for $V j j$ production $(V=W$ or $Z$ ) have been measured with the ATLAS experiment $[1,2,3]$ in several fiducial regions in the VBF topology, defined as two jets with large rapidity separation and a high dijet invariant mass. Differential cross sections have also been measured for $W$ and $Z$ production via both strong and electroweak interactions, and for electroweak interactions only. In order to more directly probe VBF production, the triple-gauge vertex has been constrained using a high- $Q^{2}$ region to increase sensitivity to new high-mass particles contributing to the effective vertex.

\section{VBF $Z$ boson production}

The purity of $Z \rightarrow \ell \ell$ production makes it an ideal early testing ground for the VBF topology. The ATLAS measurement [2] uses an integrated luminosity of $20.3 \mathrm{fb}^{-1}$ at $\sqrt{s}=8 \mathrm{TeV}$ and defines a VBF topology using two jets $\left(p_{\mathrm{T}}>50\right.$ and $\left.40 \mathrm{GeV}\right)$ with dijet invariant mass $\left(m_{j j}\right)$ larger than either $250 \mathrm{GeV}$ or $1 \mathrm{TeV}$ for low-purity and high-purity VBF regions, respectively. Differential measurements of the combined strong and electroweak production are performed in the low-purity region. Figure 1 shows the measured differential cross section as a function of $m_{j j}$ compared to the SherPa [4] and Powheg [5] plus Pythia6 [6] generators with jets produced by strong processes only (QCD) or by both strong and electroweak processes (QCD+EW). The SHERPA QCD and EW samples are merged LO $Z j j+n$-parton samples and overestimate the production of high- $m_{j j}$ events. The Powheg+Pythia6 samples are NLO, with the parton emission scale set using the MiNLO [7] procedure for the QCD sample. The PowHEG+PYTHIA6 samples model the data well.

In order to separate the pure electroweak process from processes with strong jet production, the sample is divided into events with and without an additional jet within the rapidity gap spanned by the leading two jets. Figure 1 shows that electroweak purity is increased in events without a jet in the rapidity gap. These events are used to measure the cross section of electroweak jet production in the VBF topology, while the events with an additional jet are used to derive a correction for the $m_{j j}$ modelling for strongly produced jets in SHERPA. The measured fiducial cross sections are given in Table 1.

\section{VBF $W$ boson production}

Because of the higher cross section for $W$ boson production in a VBF topology $(W j j)$, the ATLAS measurement of this process [3] at $\sqrt{s}=8 \mathrm{TeV}$ is the most precise fiducial cross section in a VBF topology and the only differential measurement of a VBF process; in addition, the fiducial 

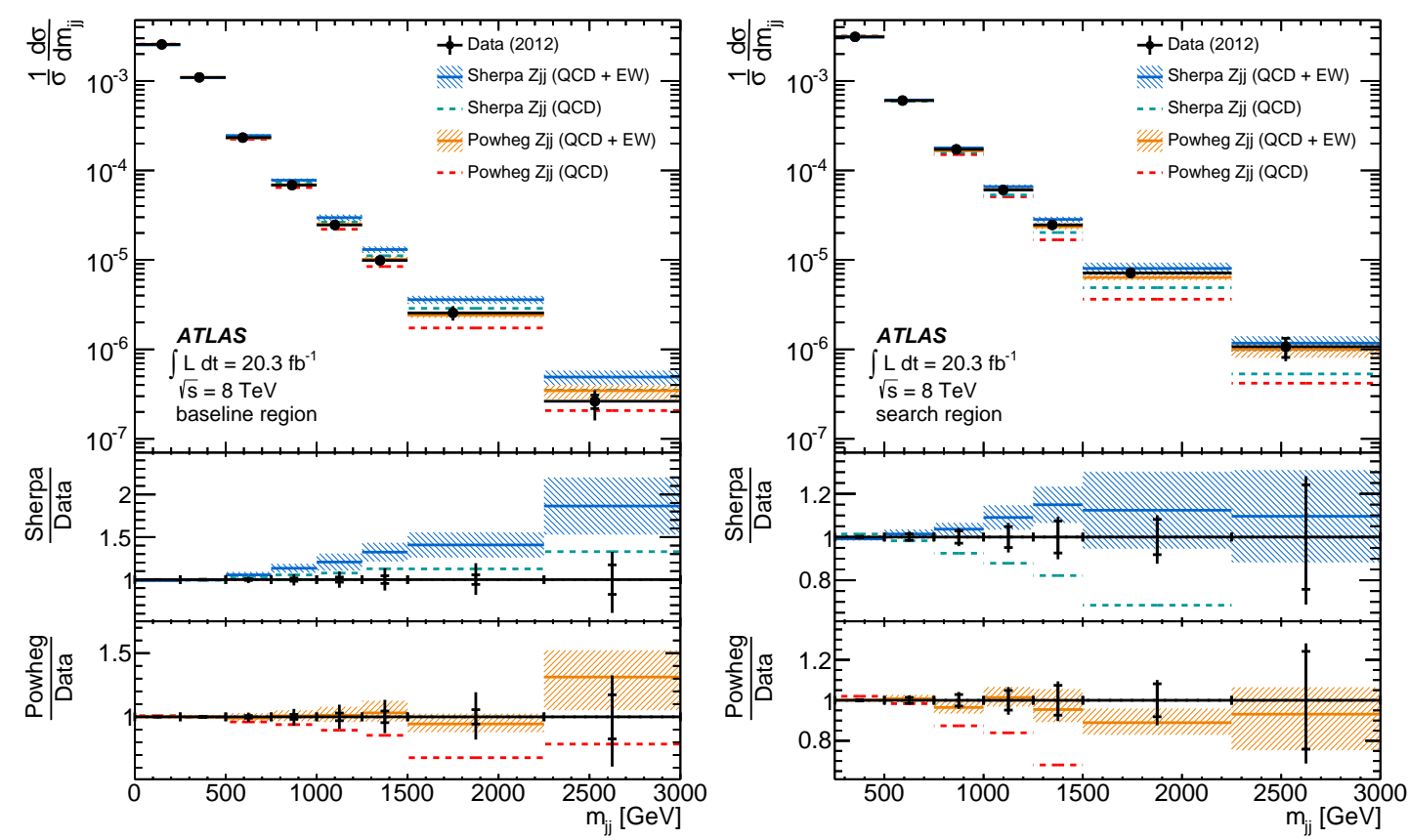

Figure 1: Unfolded differential measurement [2] of the $Z j j$ production cross section as a function of dijet invariant mass compared to SHERPA and POWHEG+PYTHIA6 predictions. The purity of electroweak jet production increases as the invariant mass increases, as this enhances the VBF $t$-channel production. The purity is small in the baseline fiducial region (left) but substantially increases with the addition of a veto on jets within the rapidity gap spanned by the leading two jets (right).

Table 1: The measured fiducial cross sections for electroweak $Z j j$ production in the VBF topology with $m_{j j}$ thresholds of $250 \mathrm{GeV}$ and $1 \mathrm{TeV}$ [2].

\begin{tabular}{l|c|c}
\hline \hline Region & Measurement [fb] & PowHEG+PythiA6 prediction [fb] \\
\hline$m_{j j}>250 \mathrm{GeV}$ & $54.7 \pm 4.6\left(\right.$ stat ${ }_{-10.4}^{+9.8}(\mathrm{sys}) \pm 1.5(\mathrm{lum})$ & $46.1 \pm 1.0$ \\
\hline$m_{j j}>1 \mathrm{TeV}$ & $10.7 \pm 0.9($ stat $) \pm 1.9(\mathrm{sys}) \pm 0.3(\mathrm{lum})$ & $9.4 \pm 0.3$ \\
\hline \hline
\end{tabular}

cross section measurement at $\sqrt{s}=7 \mathrm{TeV}$ is the only measurement of a VBF process at this energy. The event selection is similar to that of the VBF $Z$ measurement, with jet thresholds increased to 80 and $60 \mathrm{GeV}$, and the $m_{j j}$ threshold set to $500 \mathrm{GeV}$. The pure electroweak processes are separated using both a rejection on additional jets in a gap in the rapidity range of the two jets, and a requirement that the charged lepton fall within this gap.

The fiducial measurement of the pure electroweak $W j j$ process is performed with a fit to the $m_{j j}$ distribution for the cross sections for electroweakly produced jets and for strongly produced jets. To improve the modelling of the strong process in the Powheg plus Pythia8 [8] Monte Carlo, a correction is applied to the $m_{j j}$ distribution using events with a charged lepton outside the 
Table 2: The measured fiducial cross sections for electroweak $W j j$ production in the VBF topology with an $m_{j j}$ threshold of $500 \mathrm{GeV}$ at $\sqrt{s}=7$ and $8 \mathrm{TeV}[3]$.

\begin{tabular}{l|c|c}
\hline \hline$\sqrt{s}$ & Measurement [fb] & PowHEG+PythiA8 prediction [fb] \\
\hline $7 \mathrm{TeV}$ & $144 \pm 23($ stat $) \pm 23(\exp ) \pm 13($ th $)$ & $144 \pm 11$ \\
\hline $8 \mathrm{TeV}$ & $159 \pm 10($ stat $) \pm 17(\exp ) \pm 15($ th $)$ & $198 \pm 12$ \\
\hline \hline
\end{tabular}

rapidity gap used to select events for the fit. The correction is validated by applying it to events with at least one additional jet in the rapidity gap, and it reduces the uncertainty on the ratio of the measured electroweak cross section to its SM prediction from 0.18 to 0.14 at $\sqrt{s}=8 \mathrm{TeV}$. The dominant systematic uncertainties are those on the jet energy scale (0.079) and the parton distribution functions (0.052), each of which is at least as large as the statistical uncertainty (0.052). The measured fiducial electroweak cross sections at $\sqrt{s}=7$ and $8 \mathrm{TeV}$ are shown in Table 2.

The high $W j j$ event yields in $\sqrt{s}=8 \mathrm{TeV}$ data allow differential measurements of the electroweak process. Measurements are first made of combined strong and electroweak $W j j$ production to study the modelling of the strong $W j j$ background. The modelling of the jet-veto efficiency, defined as the fraction of events without a jet in the rapidity gap spanned by the leading jets, is well modelled by PowHEG+Pythia8. For an $m_{j j}$ threshold of 500 (2000) GeV, the observed efficiency is $59.6 \pm 1.4 \%(63 \pm 4 \%)$ and the predicted efficiency is $59.7 \pm 0.5 \%(63 \pm 3 \%)$. For comparison, the efficiencies in the $W j j$ process where the leading jets are produced only by the strong interaction are $56.9 \pm 0.2 \%$ and $36 \pm 3 \%$, respectively.

The differential measurements probe various kinematic regions defined by the presence of the lepton or additional jets in a rapidity gap within that of the leading jets. As in the VBF $Z$ analysis, the $m_{j j}$ distribution (Figure 2 left) is not well modelled by SHERPA but is reasonably well modelled by Powheg+Pythia8. While most distributions are modelled well by Powheg+Pythia8, the MC predicts a harder dijet $p_{\mathrm{T}}$ in the electroweak measurement region than observed in the data (Figure 2 right).

To perform differential measurements of the pure electroweak process, a region of increased purity is defined with $m_{j j}>1 \mathrm{TeV}$. The predicted $W j j$ background with strong production of jets is subtracted before unfolding the data for detector effects. Figure 3 shows that the $m_{j j}$ and leadingjet $p_{\mathrm{T}}$ distributions are reasonably well modelled by both POWHEG+PYTHIA8 and SHERPA. The differential measurements in the various regions are integrated to provide a number of fiducial cross sections, all of which agree with the POWHEG+Pythia 8 predictions to within $\approx 1.5 \sigma$.

The distribution of the leading-jet $p_{\mathrm{T}}$ is sensitive to anomalous triple-gauge couplings in the VBF production diagram. The maximum expected deviation is at high $Q^{2}$, closest to the scale of new physics. A search region is defined where the leading jet has $p_{\mathrm{T}}>600 \mathrm{GeV}$ and the dijet invariant mass is greater than $1 \mathrm{TeV}$; in this region 39 events are predicted and 30 are observed. Since there is no evidence of a non-SM contribution to this region, constraints are set on dimension-6 operators in an effective Lagrangian formalism. For the parameter sensitive only to anomalous triple-gauge couplings, $c_{W W W}$, the allowed region at $95 \%$ confidence level is $[-13,9] \Lambda^{2} / \mathrm{TeV}^{2}$. 

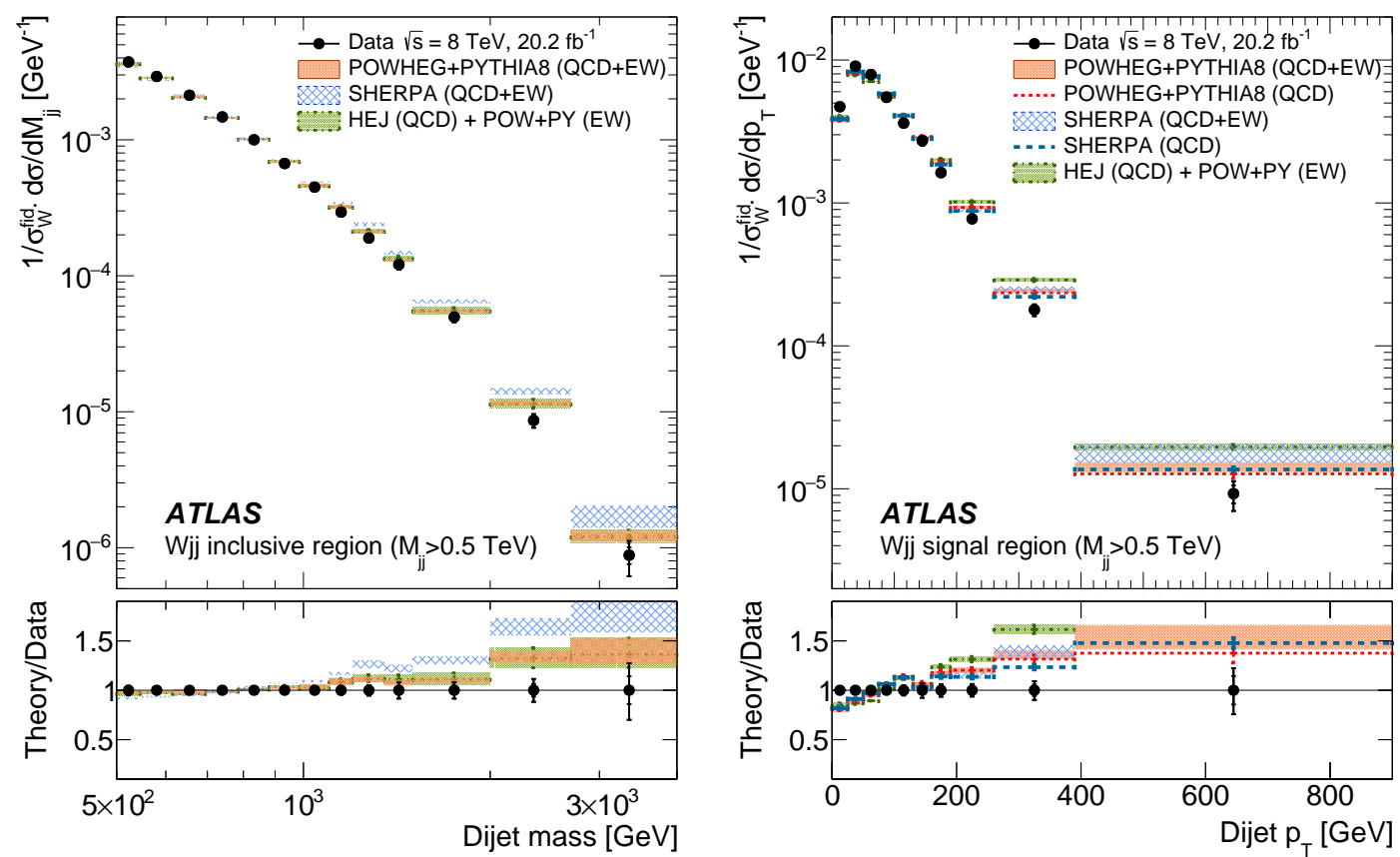

Figure 2: Unfolded differential measurements [3] of the $W j j$ production cross section as functions of dijet invariant mass (left) and dijet $p_{\mathrm{T}}$ (right), compared to SHERPA, PowHEG+PythiA8, and HEJ [9] plus POWHEG+PYTHIA8 predictions.
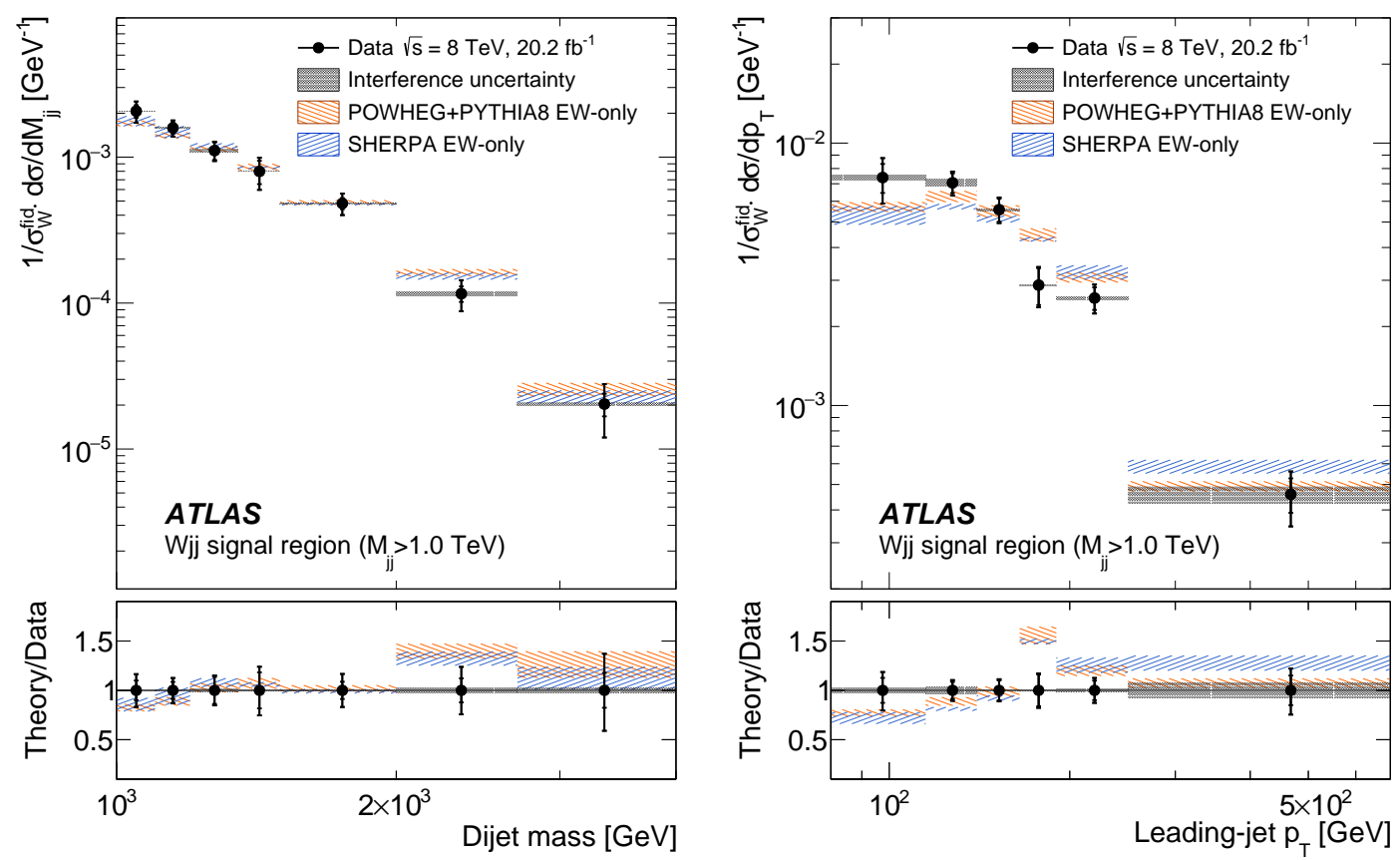

Figure 3: Unfolded differential measurements [3] of the pure electroweak $W j j$ production cross section as functions of dijet invariant mass (left) and leading jet $p_{\mathrm{T}}$ (right), compared to SHERPA and POWHEG+PYTHIA8 predictions. 


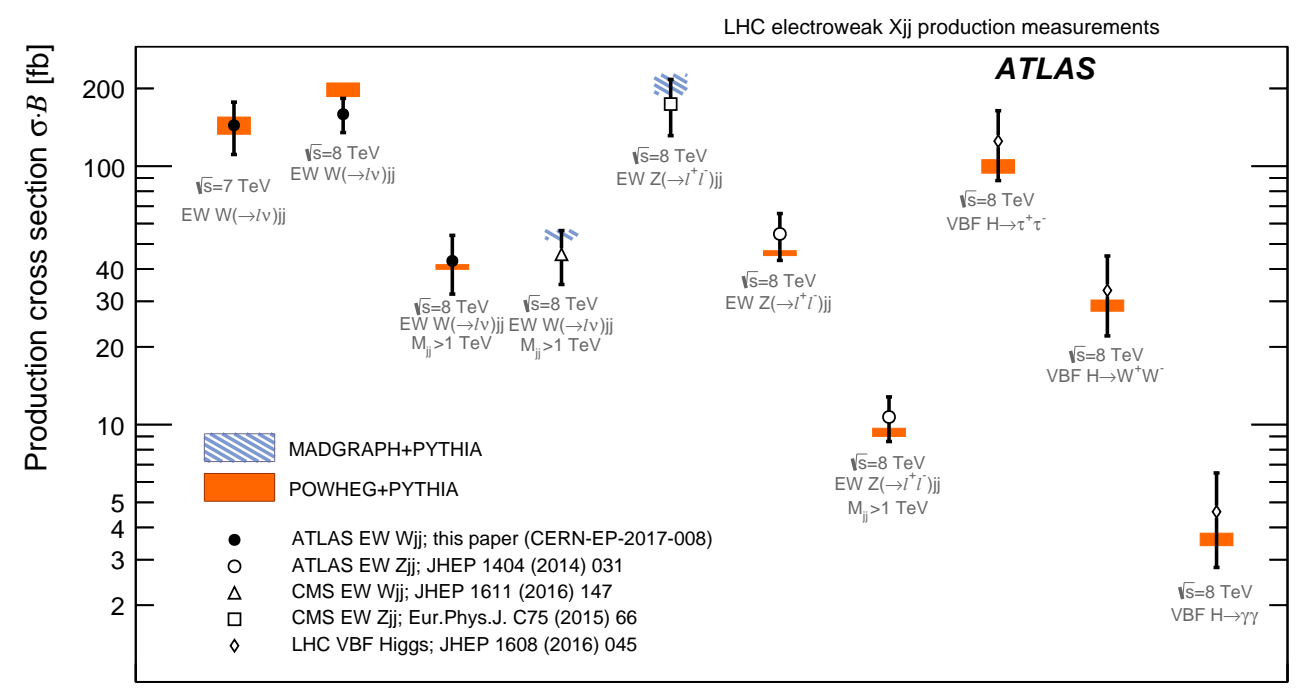

Figure 4: Measured cross sections for $V j j$ and VBF Higgs production from ATLAS, CMS, and ATLAS+CMS, compared to Monte Carlo predictions [3].

\section{Summary}

The study of single-vector-boson production in a VBF topology provides valuable information on the VBF process and its uncertainties. Many detailed studies and measurements of $W j j$ and $Z j j$ production have been performed by the ATLAS experiment at $\sqrt{s}=7$ and $8 \mathrm{TeV}$. The measurements include both the pure electroweak process and the background with strongly produced jets, which is more challenging to model. The measurements include fiducial measurements at both centre-of-mass energies and differential measurements at $\sqrt{s}=8 \mathrm{TeV}$, including differential measurements of pure electroweak $W j j$ production. Limits on anomalous gauge couplings have been derived using both the $W j j$ and $Z j j$ processes. A summary of the various $W j j, Z j j$ and VBF Higgs measurements from ATLAS and CMS are shown in Figure 4.

\section{References}

[1] ATLAS Collaboration, JINST 3, S08003 (2008).

[2] ATLAS Collaboration, JHEP 04, 031 (2014).

[3] ATLAS Collaboration, Eur. Phys. J. C 77, 474 (2017).

[4] T. Gleisberg et al., JHEP 02, 007 (2009), version 1.4.3.

[5] F. Schissler and D. Zeppenfeld, JHEP 04, 057 (2013). J. M. Campbell, R. K. Ellis, P. Nason, and G. Zanderighi, JHEP 08, 005 (2013).

[6] T. Sjöstrand, S. Mrenna, and P. Z. Skands, JHEP 05, 026 (2006).

[7] K. Hamilton, P. Nason, G. Zanderighi, JHEP 10, 155 (2012).

[8] T. Sjöstrand, S. Mrenna, and P. Z. Skands, Comput. Phys. Commun. 178, 852 (2008).

[9] J. R. Andersen, T. Hapola, J. M. Smillie, JHEP 09, 047 (2012). 